\title{
SHAPE IMPACT ON AERODYNAMIC FORCES
}

\author{
Md. Mosharrof Hossain \\ Assistant Professor, Department of Mechanical \\ Engineering \\ City University, Khagan, Savar, Dhaka-1345, \\ Bangladesh
}

\begin{abstract}
In present study, an investigation has been carried out for the unsteady turbulent flow over various type of cylinders. Reynolds- Average Navier-Stroke (RANS) equations along with SST (Shear Stress Transport) model are solved using finite element method in order to study the variation of aerodynamics forces. Linear triangular mesh elements are used for the grid generation of the computational domain. Simulation is performed at a high Reynolds number of $R e=1,00,000$ based on the free stream velocity and diameter of the cylinder. Influence of change of angle of attack on aerodynamic forces is examined. Results shows, the rectangular cylinder show maximum variation of drag and lift with the change of angle. The wake region behind the cylinders are also observed.
\end{abstract}

Keywords—cylinders, angle of attack, wake, vortex

\section{INTRODUCTION}

The flow over various types of geometric shape has become an area of great importance in fluid mechanics due to complex wake vortex formation behind the body. Flow past a cylinder is always associated with the separation of flow behind it, which incurs a large amount of energy losses. In practices, this problem has application in the field of aerodynamics engineering, engineering structures, heat exchangers, etc.

Many researches had been carried out previously on both and turbulent flow. The frequent study in this cylindrical shape is due to its simplicity in geometry and complexity in the flow characteristics. These studies have been carried out both numerically and experimentally. Eugen Dimofte et al. (2014) numerically studied turbulent flow around a circular cylinder using direct numerical simulation method (DNS) and SST $k-\omega$ model. They found that the turbulent SST $k-\omega$ model gives good prediction near the wall region than DNS model. Karabelas et al. (2012) used modified $k-\varepsilon$ model, but this model shows poor results in near the wall region. Ebrahim Shirani (2001) numerically studied flow over circular cylinder at different Reynolds number and found Mach number over 0.6, the flow parameter becomes independent of Reynolds number. Sercan Yagmur et al. (2017) experimentally and numerically studied flow characteristics around an equilateral triangle cylinder. They found that, the drag coefficient decreased with increasing Reynolds number while increasing the vortex shedding frequency. Nasaruddin Salam et al. (2014) computationally studied characteristics of fluid flowing over triangular and circular cylinder in tandem configuration. They found that the vortex is damped between the cylinders as axial distance

\author{
Muhammed Hasnain Kabir Nayeem \\ Department of Aeronautical Engineering \\ Military Institute of Science and Technology \\ Dhaka-1216, Bangladesh
}

increased. The flow around various types of cylindrical shape exhibits a remarkably complex behavior and many publications can be found in that literature (Luigi Casrassale et al. (2014), D. Arumuga Perumal et al. (2012); Roya Shademani et al. (2013); R. Gautier et al. (2013); Xu Liu et al. (2020); J. F. Derakhshandeh et al. (2017)). The present study is devoted to the study of flow past circular, rectangular, square and triangular cylinder.

\section{Computational MODEL}

The dimension of the cylinders are, for circular cylinder the diameter is $50 \mathrm{~mm}$, for rectangular cylinder $25 \mathrm{~mm} * 100 \mathrm{~mm}$, for square cylinder and equilateral triangular cylinder the length of each edges are $50 \mathrm{~mm}$. A rectangular domain of dimension $11 \mathrm{D} * 38 \mathrm{D}$ where, $\mathrm{D}$ is the diameter of the circular cylinder is considered in the present study shown in fig-1. The Cartesian coordinate system is adopted and the origin of the domain is set at $(0,0)$, the cylinders were placed in the origin.

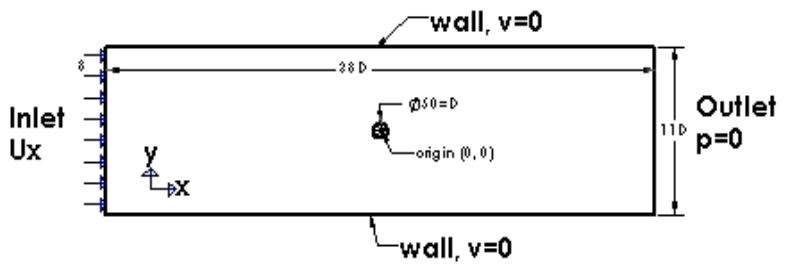

Figure 1: Schematic diagram of the computational domain

\section{MATHEMATICAL MODEL}

The fluid flow problem is defined by the law of conservation of mass, momentum and energy. These laws are expressed in terms of partial differential equations which are discretized with a finite element based technique. The mathematical model is draw up considering incompressible, one phase, fixed domain Newtonian fluid flow over the cylinder. From the law of conservation of mass comes the continuity equation,

$$
\frac{\partial p}{\partial t}+\frac{d\left(p v_{x}\right)}{d x}+\frac{d\left(p v_{y}\right)}{d y}+\frac{d\left(p v_{z}\right)}{d z}=0
$$

Where, $v_{x}, v_{y}, v_{z}$ are the components of the velocity vector in the global Cartesian coordinate $\mathrm{x}, \mathrm{y}$ and $\mathrm{z}$ direction respectively. In a Newtonian fluid, the relationship between the stress and rate of deformation of fluid is,

$$
\tau_{i j}=-\rho \delta_{i j}+\mu\left(\frac{d_{u i}}{d_{x j}}+\frac{d_{u j}}{d_{x i}}\right)+\delta_{i j} \lambda \frac{d_{u i}}{d_{x}}
$$




\section{International Journal of Engineering Applied Sciences and Technology, 2020 \\ Vol. 5, Issue 1, ISSN No. 2455-2143, Pages 26-29 \\ Published Online May 2020 in IJEAST (http://www.ijeast.com)}

Where, $\tau_{i j}$ is the stress tensor, $u_{i}$ is orthogonal velocities, $\mu$ is the dynamics viscosity, $\lambda$ is second coefficient of viscosity.

In present study, SST turbulent model is used. Since it uniting the predominant behavior of $k-\omega$ model in near wall zone with robustness of the $k-\varepsilon$ model (W. P. Jones, 1972). The model equation are expressed in term of turbulence kinematic energy, $\mathrm{k}$ and specific rate of dissipation, $\omega$, which are given as follows.

$\rho \frac{\partial k}{\partial t}+\rho u . \nabla k=P-\rho \beta_{0}{ }^{*} k \omega+\nabla \cdot\left[\left(\mu+\sigma_{k} \mu_{t}\right) \nabla k\right]$

$\rho \frac{\partial \omega}{\partial t}+\rho u . \nabla \omega=\frac{\rho \gamma}{\mu_{t}} P-\rho \beta \omega^{2}+\nabla \cdot\left[\left(\mu+\sigma_{\omega} \mu_{t}\right) \nabla \omega\right]+$ $2\left(1-f_{v 1}\right) \frac{\rho \sigma_{\omega 2}}{\omega} \nabla \omega . \nabla k$

(4)

Where, $P$ is a production limiter cased in the model to prevent the turbulent built-up in stagnation region.

The governing parameter of the present study is Reynolds number $(\mathrm{Re})$ defined as follow,

$$
R e=\frac{\rho U_{\infty} D}{\mu}
$$

Where, $\rho$ is the density of the fluid, $U_{\infty}$ is the flow velocity, $\mu$ is dynamic viscosity of the fluid.

\section{NUMERICAL MODEL}

Finite-element method is used to carry out the numerical simulation of the present problem. At first, the whole computational domain is discretized into a finite number of triangular mesh as shown in fig-2. Extremely finer mesh is applied around the cylinder, since the changes of velocity and pressure around these cylinder is immense.

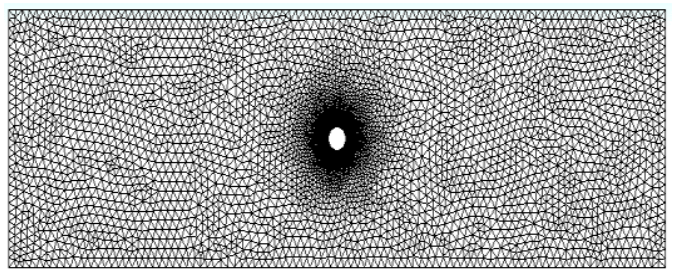

Figure 2: Mesh structure of the computational domain

\section{RESULT AND DISCUSSION}

The aerodynamic performance in terms of drag and lift coefficient is observed. Moreover, the time averaged streamline and velocity filed are visualized in order to identify different flow characteristics.

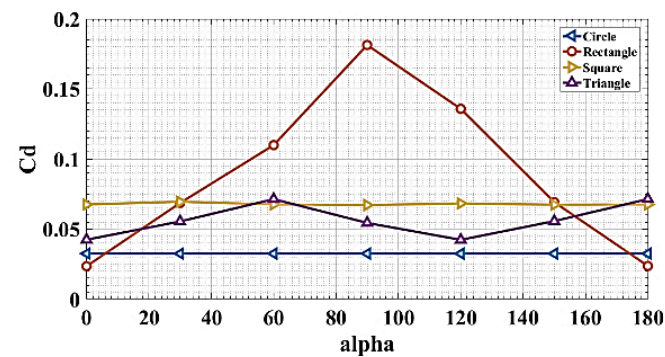

Figure 3: Variation of $C_{d}$ with angle of attack alpha $(\alpha)$

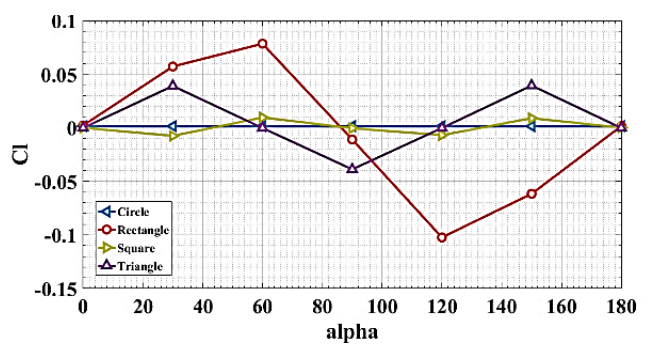

Figure 4: Variation of $C_{l}$ with angle of attack alpha $(\alpha)$

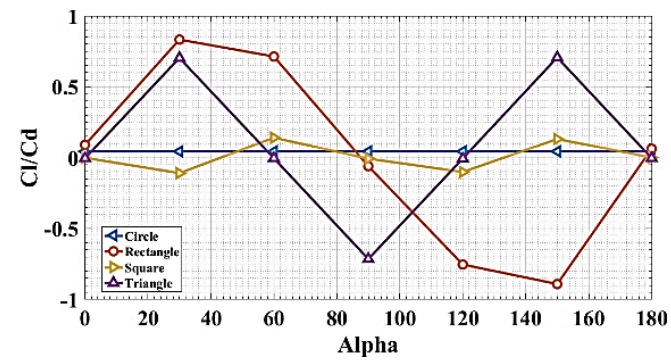

Figure 5: Variation of $C_{l} / C_{d}$ with angle of attack alpha $(\alpha)$

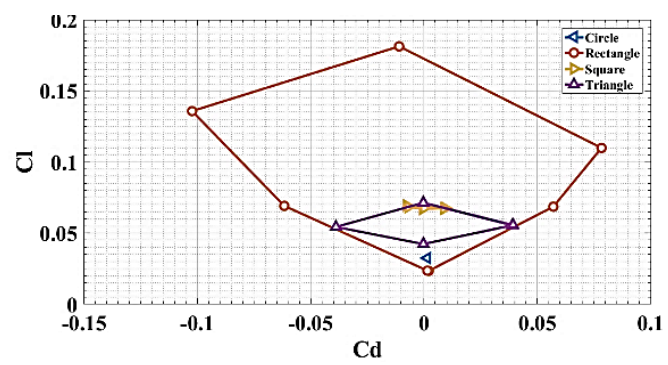

Figure 6: Variation of $C_{l}$ with $C_{d}$

Figure- 3 and fig- 4 shows the variation of $C_{d}$ and $C_{l}$ of the various cylindrical shape with the change of angle of attack $\alpha$, where $\alpha$ is the angle of attack(AOA) which is the angle between the oncoming air or relative wind and the reference line of the body. It is seen that, for the circular cylinder the lift and drag coefficient remain constant with the change of angle of attack. In the case of rectangular, square and triangular cylinder, there is a change on $C_{l}$ and $C_{d}$ found with the change of angle of attack. For square cylinder, a little variation occurs on aerodynamic forces with the change of AOA. For triangular cylinder, initially with the change of AOA the drag increases, and at $\alpha=60^{\circ}$ the maximum drag observed and then it gradually decreases and from $\alpha=90^{\circ}$ we see the similar cycle repetition. For the lift curve, the maximum lift coefficient observed at the $\alpha=30^{\circ}$ and $\alpha=150^{\circ}$ and minimum lift observed at $\alpha=90^{\circ}$.

For the rectangular cylinder, maximum fluctuation of drag and lift coefficient with the change of AOA is found. For rectangular cylinder with the change of AOA the drag coefficient increases and at $\alpha=90^{\circ}$ it reaches the maxima and then gradually started to decrease with the increase in AOA. For lift coefficient the maximum lift is observed at $\alpha=$ $60^{\circ}$ minimum lift coefficient observed at $\alpha=120^{\circ}$. 


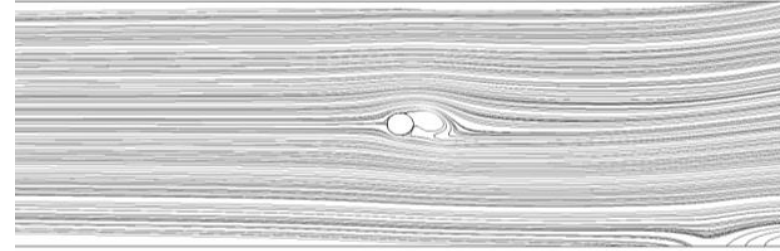

Figure 7: Time-mean streamlines for circular cylinder

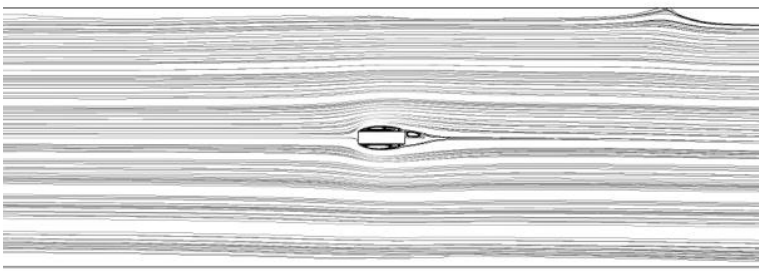

Figure 8: Time-mean streamlines for rectangular cylinder at $\alpha=0^{0}$

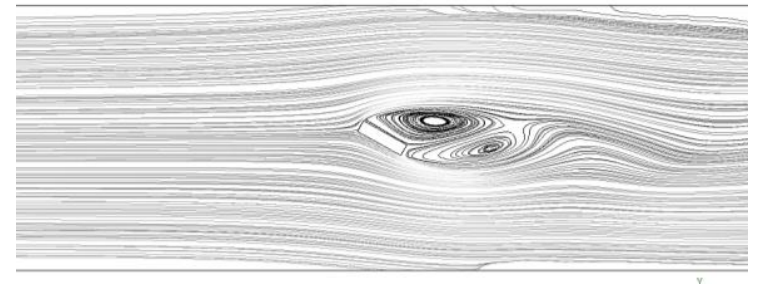

Figure 9: Time-mean streamlines for rectangular cylinder at $\alpha=30^{\circ}$

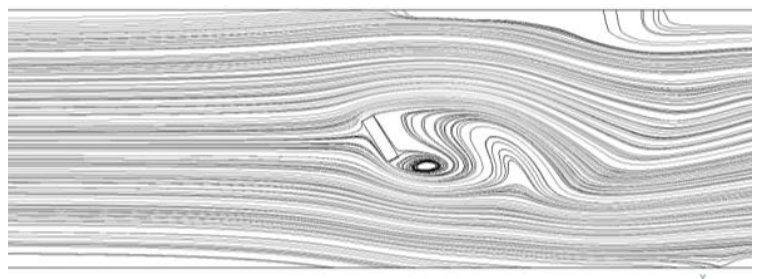

Figure 10: Time-mean streamlines for rectangular cylinder at $\alpha=60^{\circ}$

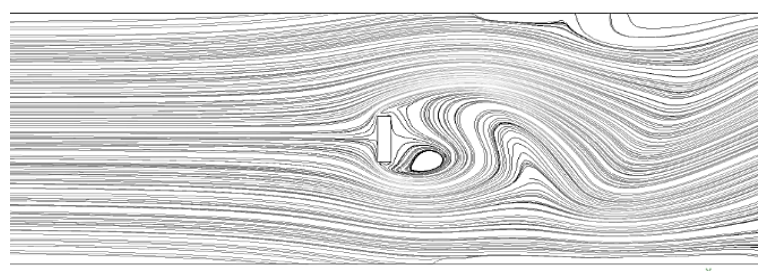

Figure 11: Time-mean streamlines for rectangular cylinder at $\alpha=90^{\circ}$

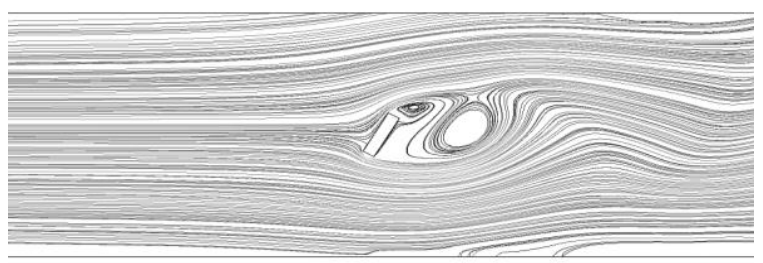

Figure 12: Time-mean streamlines for rectangular cylinder at

$$
\alpha=120^{\circ}
$$

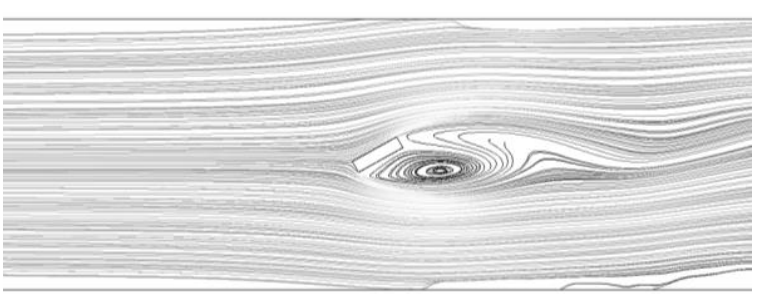

Figure 13: Time-mean streamlines for rectangular cylinder at $\alpha=150^{0}$

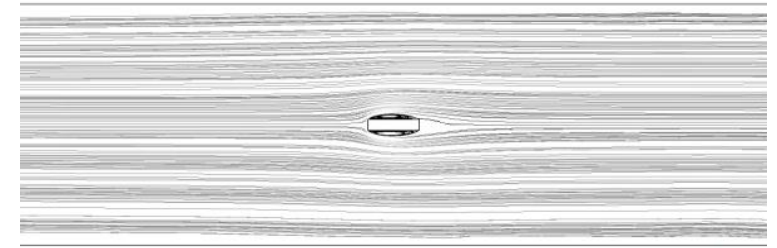

Figure 14: Time-mean streamlines for rectangular cylinder at $\alpha=180^{\circ}$

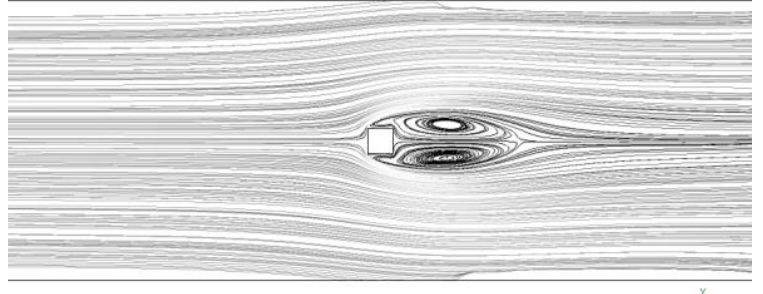

Figure 15: Time-mean streamlines for square cylinder at $\alpha=$ $0^{0}$

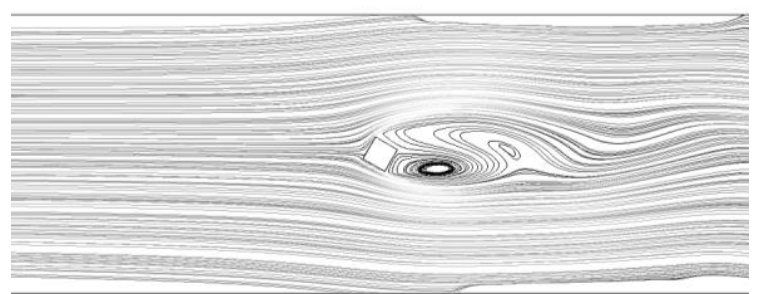

Figure 16: Time-mean streamlines for square cylinder at $\alpha=$ $30^{0}$

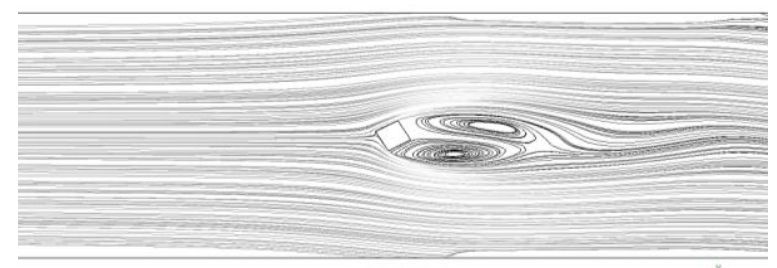

Figure 17: Time-mean streamlines for square cylinder at $\alpha=$ $60^{\circ}$

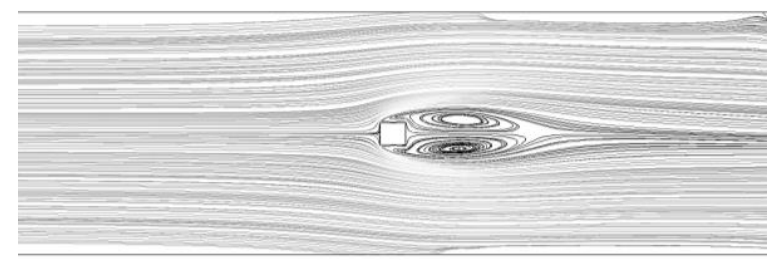

Figure 18: Time-mean streamlines for square cylinder at $\alpha=$ $90^{\circ}$ 


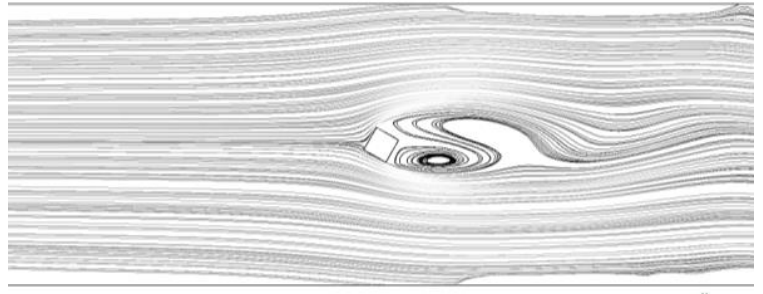

Figure 19: Time-mean streamlines for square cylinder at $\alpha=$ $120^{\circ}$

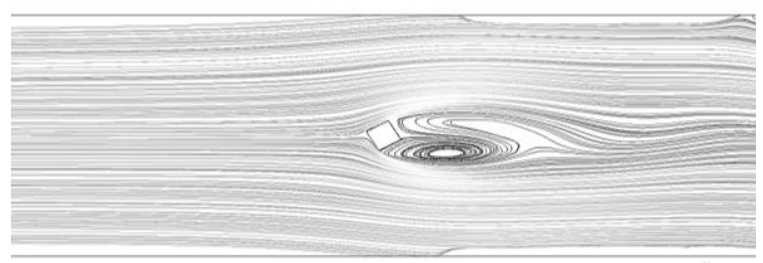

Figure 20: Time-mean streamlines for square cylinder at $\alpha=$ $150^{0}$

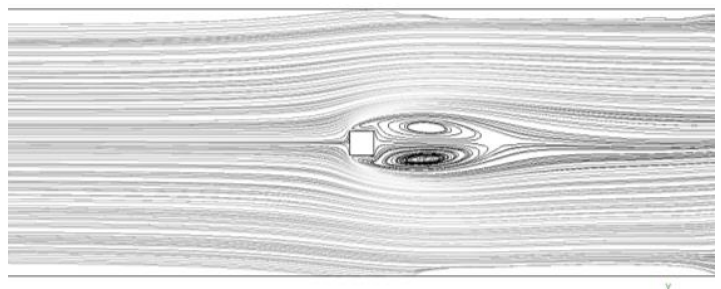

Figure 21: Time-mean streamlines for square cylinder at $\alpha=$ $180^{\circ}$

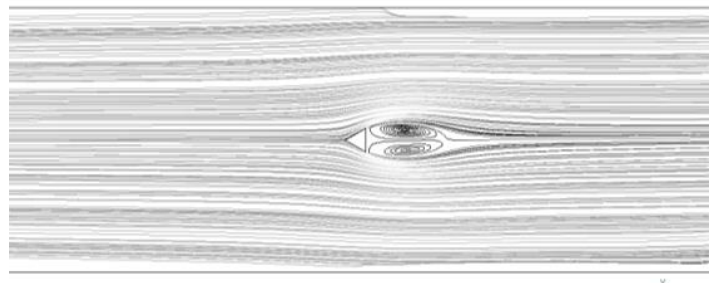

Figure 22: Time-mean streamlines for triangular cylinder at $\alpha=0^{0}$

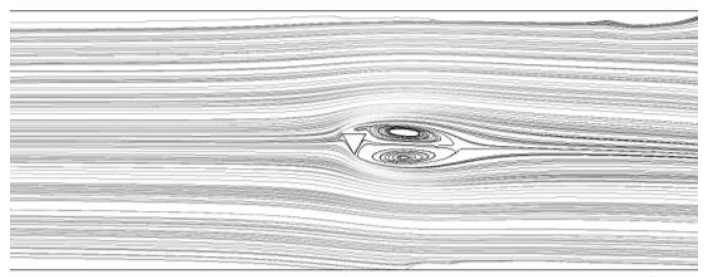

Figure 23: Time-mean streamlines for triangular cylinder at $\alpha=30^{\circ}$

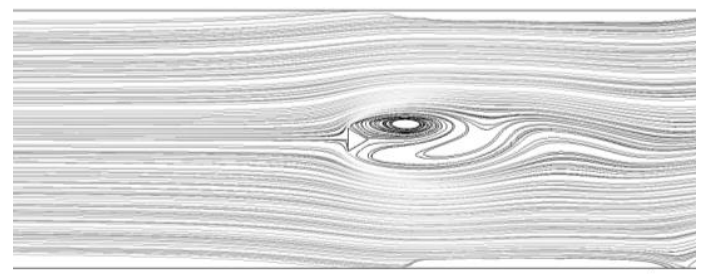

Figure 24: Time-mean streamlines for triangular cylinder at $\alpha=60^{\circ}$

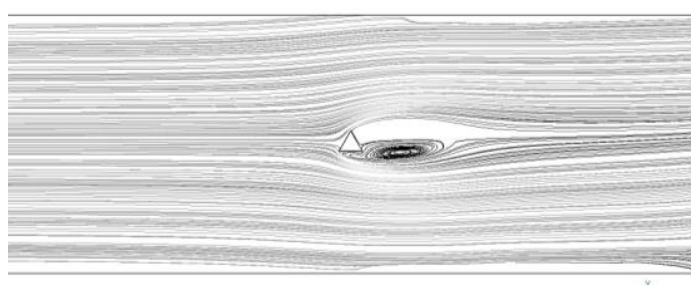

Figure 25: Time-mean streamlines for triangular cylinder at $\alpha=90^{\circ}$

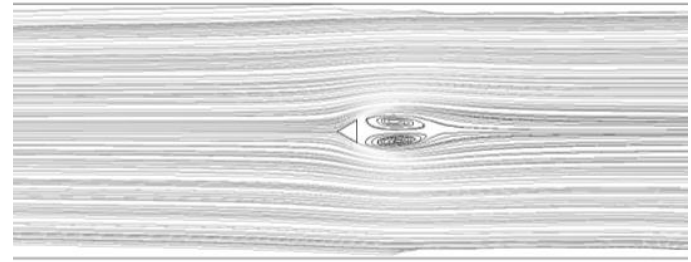

Figure 26: Time-mean streamlines for triangular cylinder at $\alpha=120^{0}$

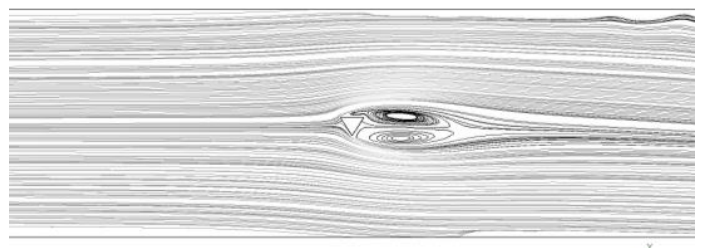

Figure 27: Time-mean streamlines for triangular cylinder at $\alpha=150^{\circ}$

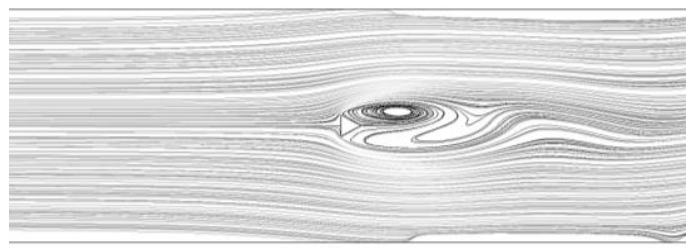

Figure 28: Time-mean streamlines for triangular cylinder at $\alpha=180^{\circ}$

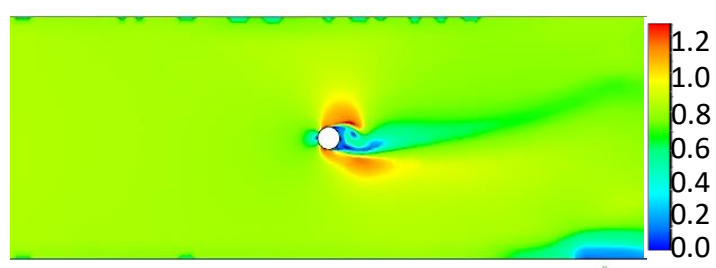

Figure 29: Velocity field contour for circular cylinder

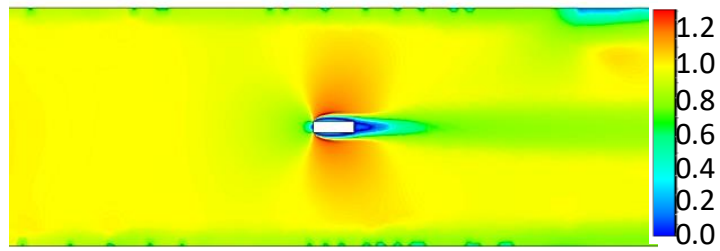

Figure 30: Velocity field contour for rectangular cylinder at $\alpha=0^{0}$

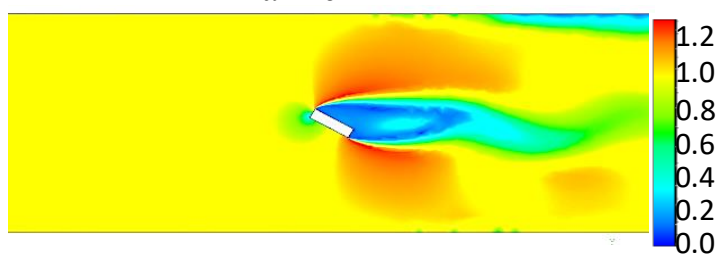




\section{International Journal of Engineering Applied Sciences and Technology, 2020 \\ Vol. 5, Issue 1, ISSN No. 2455-2143, Pages 26-29 \\ Published Online May 2020 in IJEAST (http://www.ijeast.com)}

Figure 31: Velocity field contour for rectangular cylinder at $\alpha=30^{\circ}$

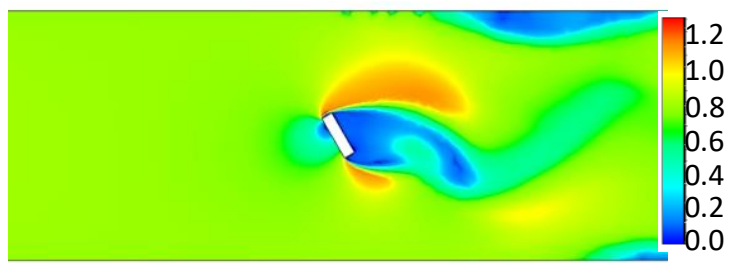

Figure 32: Velocity field contour for rectangular cylinder at $\alpha=60^{\circ}$

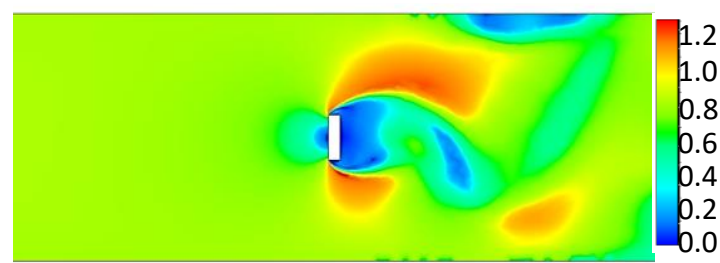

Figure 33: Velocity field contour for rectangular cylinder at $\alpha=90^{\circ}$

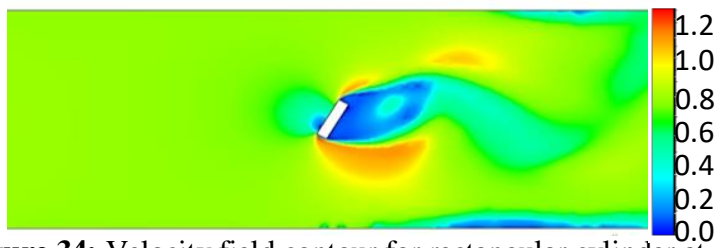

Figure 34: Velocity field contour for rectangular cylinder at $\alpha=120^{\circ}$

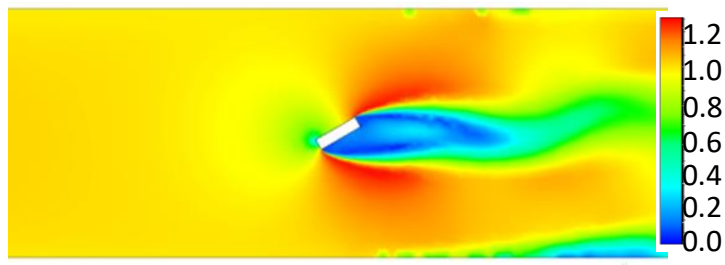

Figure 35: Velocity field contour for rectangular cylinder at $\alpha=150^{\circ}$

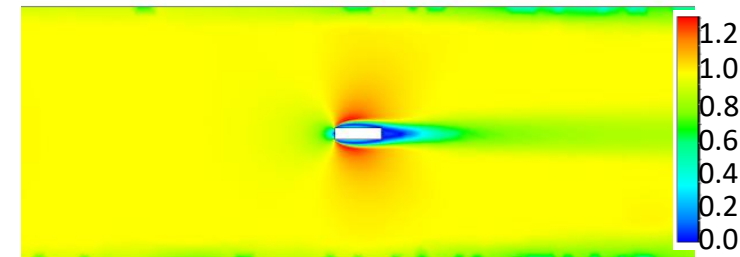

Figure 36: Velocity field contour for rectangular cylinder at $\alpha=180^{\circ}$

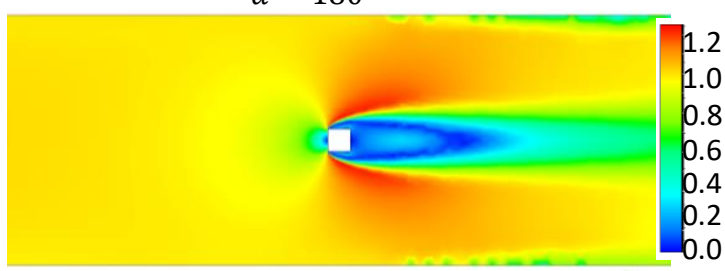

Figure 37: Velocity field contour for square cylinder at $\alpha=0^{0}$

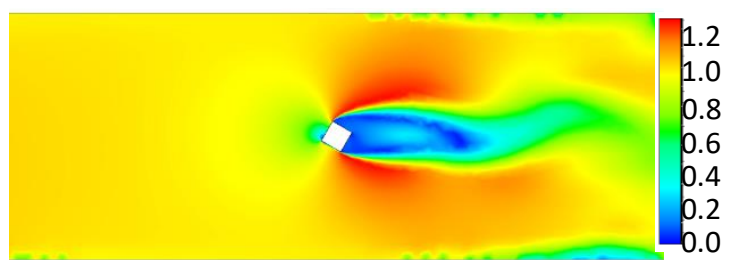

Figure 38: Velocity field contour for square cylinder at $\alpha=$ $30^{0}$

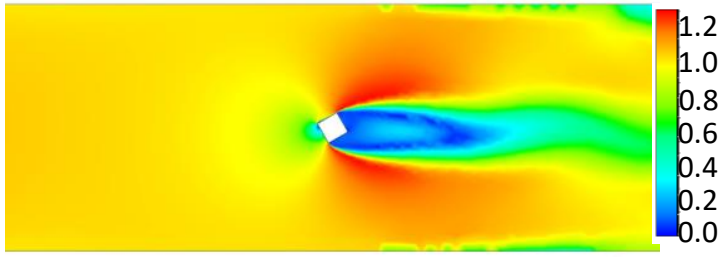

Figure 39: Velocity field contour for square cylinder at $\alpha=$ $60^{\circ}$

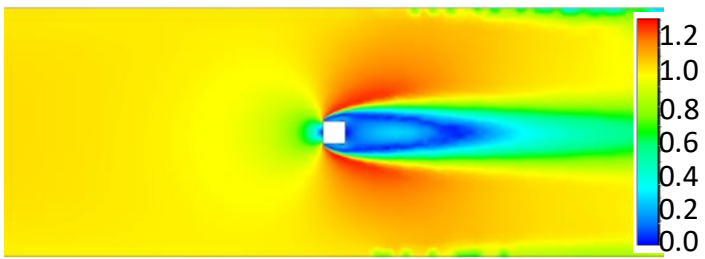

Figure 40: Velocity field contour for square cylinder at $\alpha=$ $90^{0}$

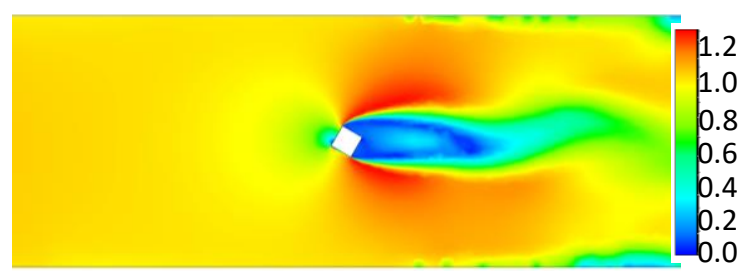

Figure 41: Velocity field contour for square cylinder at $\alpha=$ $120^{0}$

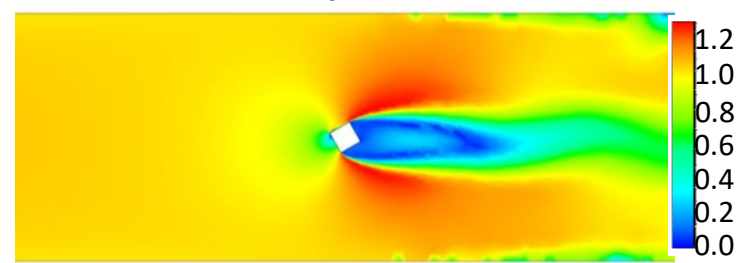

Figure 42: Velocity field contour for square cylinder at $\alpha=$ $150^{0}$

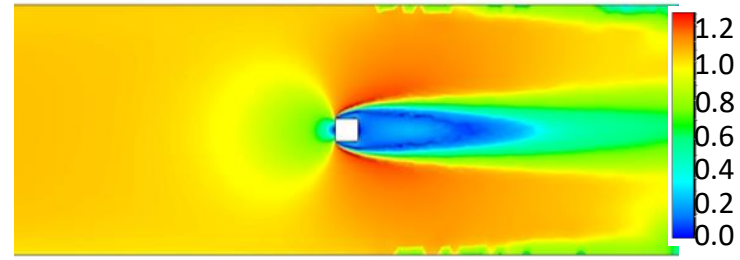

Figure 43: Velocity field contour for square cylinder at $\alpha=$ $180^{\circ}$

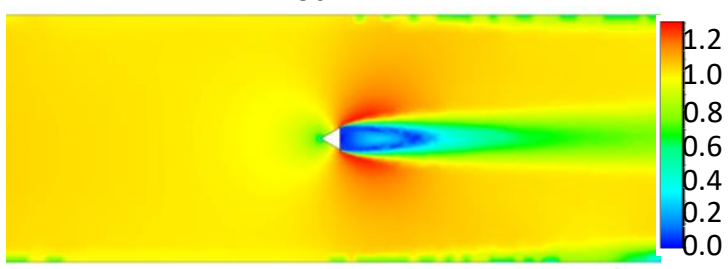

Figure 44: Velocity field contour for triangular cylinder at $\alpha=$ $0^{0}$ 


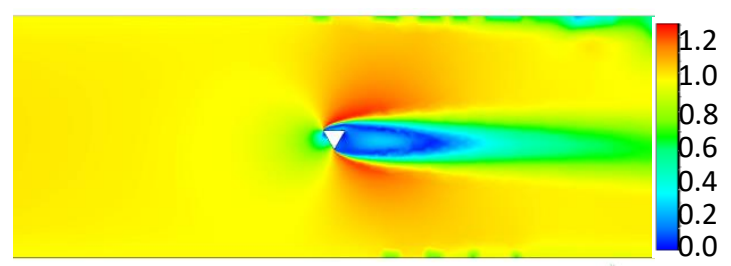

Figure 45: Velocity field contour for triangular cylinder at $\alpha=$ $30^{0}$

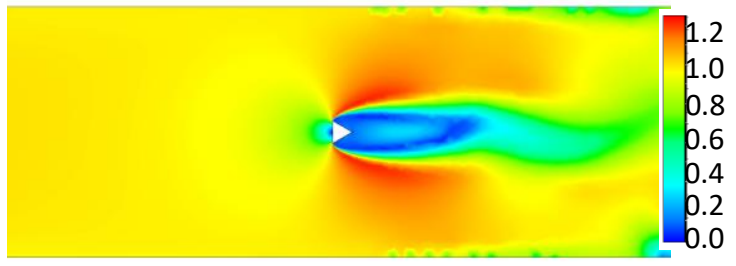

Figure 46: Velocity field contour for triangular cylinder at $\alpha=$ $60^{0}$

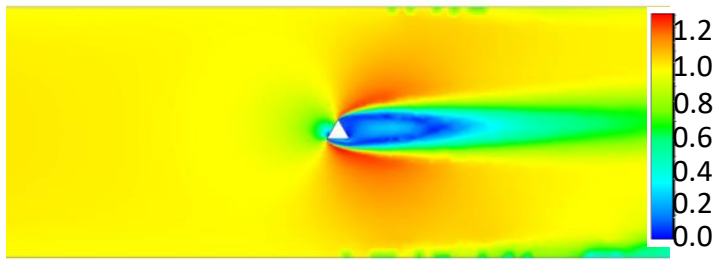

Figure 47: Velocity field contour for triangular cylinder at $\alpha=$ $90^{\circ}$

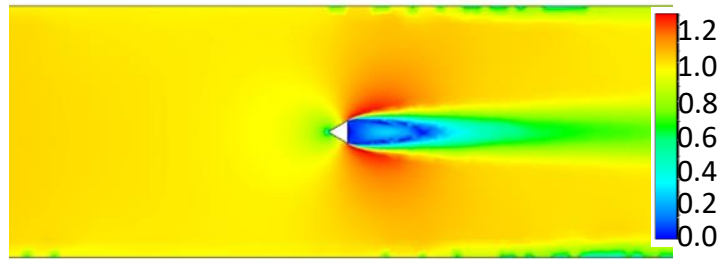

Figure 48: Velocity field contour for triangular cylinder at $\alpha=$ $120^{\circ}$

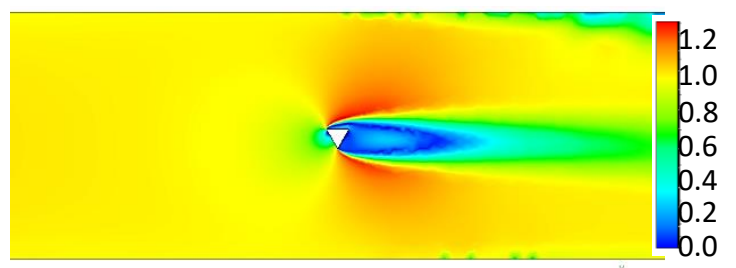

Figure 49: Velocity field contour for triangular cylinder at $\alpha=$ $150^{\circ}$

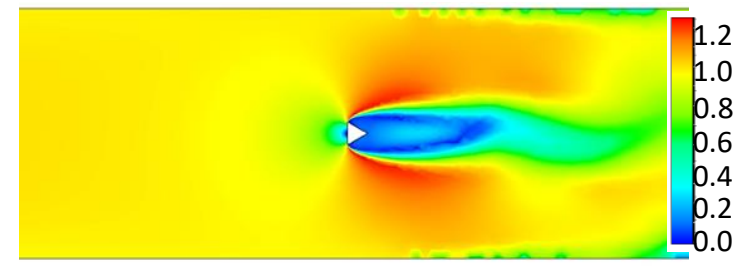

Figure 50: Velocity field contour for triangular cylinder at $\alpha=$ $180^{\circ}$

Now, attention is paid to examine the wake formation due to the presence of body. Figure- 7 to fig- 28 shows the timemean streamlines for the circular, rectangular, square and triangular cylinder. The rectangular cylinder form wide spread strong vortex at $\alpha=90^{\circ}$. From fig-29 to fig-50, shows the velocity field contours for the cylinders. For $\alpha=0^{0}$ there is symmetric velocity contour found on the presence of the body. As the angle changes the fluctuation found on the vortex formation on the wake. For rectangular cylinder at $\alpha=90^{\circ}$ strong vortices formation found, whereas for square cylinder at $\alpha=0^{\circ}, 90^{\circ}$ and $180^{\circ}$ and for triangular cylinder at $\alpha=$ $60^{\circ}, 180^{\circ}$ strong vortex formation is observed.

\section{CONCLUSION}

The present study is numerically presented the change of aerodynamics forces with the change of angle of attack of circular, rectangular, square and triangular cylinder. In can be conclude that, the wake vortex has a strong effect on the boundary layer development on the body. For the rectangular cylinder, the fluctuation of lift and drag coefficient is more prominent that than any other cylindrical shape.

\section{REFERENCES}

1. Eugen Dimofte, Florin Popescu, Ion V. Ion (2014). Numerical Modeling of Turbulent Flow Around a Circular Cylinder. The Annals of DUNAREA DE JOS University of Galati, Fascicle IV Refrigerating Technique, Internal Combustion Engines, Boilers and Turbines. ISSN 1221-4558

2. S. J. Karabelas, B. C. Koumroglou, C. D. Argyropoulos and N. C. Markatos (2012). High Reynolds Number Turbulent Flow Past A Rotating Cylinder. Applied Mathematical Modelling, Volume 36, Issue 1, ( pp. 379-398)

3. Ebrahim Shirani (2001).Compressible Flow Around A Circular Cylinder. Pakistan Journal of Applied Sciences, 1 (4), (pp. 472-476)

4. Sercan Yagmur, Sercan Dogan, Muharrem Hilmi Aksoy, Ilker Goktepeli, Muammer Ozgoren. (2017). Comparison Of Flow Characterisitics Around An Equilateral Triangular Cylinder Via PIV And Large Eddy Simulation Methods. Flow Measurement and Instrumentation, Volume 55, (pp. 23-36)

5. Nasaruddin Salam, I. N. G. Wardana, Slamet Wahyudi, Denny Widhiyanuriyawan (2014). Fluid Flow Through Triangular And Square Cylinders. Australian Journal of Basic and Applied Sciences, 8(2), (pp. 193-200)

6. Luigi Carassale, Andrea Freda, Michela MarreBrunenghi (2014). Experimental Investigation On The Aerodynamics Behavior Of Square Cylinders With Rounded Corners. Journal of Fluids and Structures, volume 44, (pp. 195-204)

7. D. Arumuga Perumal, Gundavarapu V.S. kumar, Anoop K. Dass (2012). Numerical Simulation Of Viscous Flow Over A Square Cylinder Using Lattice Boltzmann Method. ISRN Mathematical Physics

8. Roya Shademani, Parviz Ghadimi, Rahim Zamanian, Abbas Dashtimanesh. (2013). Assessment Of Air Flow Over An Equilateral 


\section{Published Online May 2020 in IJEAST (http://www.ijeast.com)}

Triangular Obstacle In A Horizontal Channel Using FVM. Journal of Mathematical Sciences and Applications, Volume 1, No 1, (pp. 12-16)

9. R. Gautier, D. Biau, E. Lamballais (2013). A refernece solution of the flow over a circular cylinder at $\mathrm{Re}=40$. Computers \& Fluids, Volume 75, (pp. 103-111)

10. Xu Liu, Nan Gui, Hao Wu, Xingtuan Yang, Jiyuan Tu, Shengyao Jiang (2020). Numerical Simulation Of Flow Past A Triangular Prism With FluidStructure Interaction. Engineering Applications of
Computational Fluid Mechanics, Volume 14, No 1, (pp. 462-476)

11. J. F. Derakhshandeh, Md. Mahbub Alam (2017). Flow Structures Around A Rectangular Cylinder In The Vicinity Of A Wall. Advances in Structural Engineering and Mechanics

12. W. P. Jones, B. E. Launder (1972). The Prediction Of Laminarization With A Two-Equation Model Of Turbulence. International Journal of Heat and Mass Transfer, volume 15, Issue 2, (pp. 301-314) 